\title{
The effect of prunes on stool output, whole gut transit time and gastrointestinal symptoms: a randomised controlled trial
}

This abstract was presented as the Public Health Nutrition Theme highlight.

Prunes (dried plums) are perceived to help maintain healthy bowel function ${ }^{(1)}$ and preliminary research suggests they improve stool frequency and consistency in mild constipation ${ }^{(2)}$. The high fibre and sorbitol content may aid in laxation ${ }^{(3)}$, however the effects of prunes on gastrointestinal (GI) health are not well researched and mechanisms of action are unclear.

We conducted a three-arm, parallel group, randomised controlled trial to investigate the dose dependent effect of prunes on GI outcomes. 120 healthy people with $3-6$ stools/wk (mean age 35 years, $60 \%$ female) were randomised to high-dose prunes (120 g/d $+300 \mathrm{ml}$ water/d), low-dose prunes $(80 \mathrm{~g} / \mathrm{d}+300 \mathrm{ml}$ water/d) or control $(300 \mathrm{ml}$ water/d) for 4 weeks. The primary outcome was stool weight, assessed by 7-day total stool collection. Other outcomes included stool frequency and consistency (stool diary), whole gut transit time (WGTT, radio-opaque markers) and GI symptoms (GI symptom rating scale). Outcome assessors were blinded to group assignment. Intervention effects were compared using analysis of covariance with baseline measurements as covariates, or Kruskal-Wallis test for non-parametric variables.

In the per-protocol analysis $(n=104)$, compared with control, $120 \mathrm{~g}$ prunes increased stool weight and $80 \mathrm{~g}$ prunes increased the number of bowel movements (BMs). $80 \mathrm{~g}$ prunes also increased spontaneous BMs, but prunes had no effect on complete BMs, complete spontaneous BMs, WGTT, stool consistency, stool water and stool $\mathrm{pH}$.

\begin{tabular}{|c|c|c|c|c|c|c|c|c|c|c|c|c|}
\hline & \multicolumn{4}{|c|}{ Control $(n=35)$} & \multicolumn{4}{|c|}{$80 \mathrm{~g} / \mathrm{d}$ prunes $(n=37)$} & \multicolumn{4}{|c|}{$120 \mathrm{~g} / \mathrm{d}$ prunes $(n=32)$} \\
\hline & \multicolumn{2}{|c|}{ Baseline } & \multicolumn{2}{|c|}{ Intervention } & \multicolumn{2}{|c|}{ Baseline } & \multicolumn{2}{|c|}{ Intervention } & \multicolumn{2}{|c|}{ Baseline } & \multicolumn{2}{|c|}{ Intervention } \\
\hline & Mean & $\overline{\mathrm{SD}}$ & Mean & $\overline{\mathrm{SD}}$ & Mean & $\overline{\mathrm{SD}}$ & Mean & $\overline{\mathrm{SD}}$ & Mean & $\overline{\mathrm{SD}}$ & $\overline{\text { Mean }}$ & $\mathrm{SD}$ \\
\hline Stool weight $(\mathrm{g} / \mathrm{d})$ & 110 & $43 \cdot 0$ & 109 & 61.4 & 90 & $45 \cdot 1$ & 114 & $70 \cdot 5$ & 99 & 51.6 & $135^{*}$ & 71.4 \\
\hline BMs (wk) & $5 \cdot 5$ & 1.4 & $5 \cdot 5$ & $2 \cdot 2$ & $5 \cdot 2$ & $2 \cdot 1$ & $7 \cdot 0 * *$ & $3 \cdot 9$ & $5 \cdot 0$ & 1.7 & $5 \cdot 7$ & $1 \cdot 6$ \\
\hline SBMs (wk) & $5 \cdot 4$ & 1.4 & $5 \cdot 5$ & $2 \cdot 2$ & $5 \cdot 1$ & $2 \cdot 0$ & $7 \cdot 0^{* * *}$ & $3 \cdot 9$ & $5 \cdot 0$ & 1.7 & $5 \cdot 5$ & 1.5 \\
\hline WGTT (hrs) & $43 \cdot 4$ & $27 \cdot 4$ & $36 \cdot 3$ & 28.9 & $41 \cdot 0$ & $29 \cdot 1$ & $32 \cdot 8$ & $27 \cdot 3$ & $46 \cdot 5$ & $30 \cdot 5$ & $39 \cdot 5$ & 29.4 \\
\hline BSC mean score & $4 \cdot 0$ & $1 \cdot 2$ & $3 \cdot 9$ & $1 \cdot 1$ & $3 \cdot 5$ & $1 \cdot 1$ & $3 \cdot 8$ & $1 \cdot 2$ & $3 \cdot 3$ & 1.9 & $3 \cdot 6$ & $1 \cdot 0$ \\
\hline Stool water $(\%)$ & $73 \cdot 2$ & $7 \cdot 2$ & 73.7 & $7 \cdot 1$ & $72 \cdot 0$ & $6 \cdot 4$ & 73.9 & $7 \cdot 2$ & $71 \cdot 0$ & $5 \cdot 2$ & $73 \cdot 2$ & $7 \cdot 2$ \\
\hline Stool $\mathrm{pH}$ & $6 \cdot 8$ & 0.5 & $6 \cdot 7$ & 0.5 & $6 \cdot 7$ & 0.6 & $6 \cdot 6$ & 0.5 & 6.9 & $0 \cdot 4$ & $6 \cdot 6$ & 0.6 \\
\hline
\end{tabular}

WGTT, whole gut transit time; BMs, bowel movements; BSC, Bristol Stool chart (1-7)

Means are significantly different from control (ANCOVA with baseline as covariate): * P $0.035 * * P 0.027$ *** P 0.032

Of 14 GI symptoms recorded, incidence of flatulence was higher in the $120 \mathrm{~g}$ group (4.9 (SD 2.2) d/wk) and $80 \mathrm{~g}$ group (4.8 (SD 2.6 ) $\mathrm{d} / \mathrm{wk}$ ), compared to control (0.5 (SD 0.6) d/wk, P 0.009) and severity of flatulence was higher in the $120 \mathrm{~g}$ group (1.1 (SD 0.7)) and $80 \mathrm{~g}$ group (1.0 (SD 0.7)) compared with control (0.5 (SD 0.6), P 0.001) although actual severity was relatively low $(0=$ absent, $1=$ mild, $2=$ moderate, 3 = severe). Incidence of acid reflux was higher in the $120 \mathrm{~g}$ group, compared to control (0.7 (SD 1.4) d/wk vs 0.1 (SD 0.2) $\mathrm{d} / \mathrm{wk}, \mathrm{P} 0 \cdot 02)$ as was its severity $(0 \cdot 1$ (SD $0 \cdot 2)$ vs $0 \cdot 0$ (SD 0.0), P 0.02).

In healthy individuals, prunes seem well tolerated and significantly increase stool weight and bowel movements, but do not decrease WGTT. Stool weight is inversely related to colon cancer risk ${ }^{(4)}$. Therefore prunes have potential health benefits in the UK where average stool weight is low ${ }^{(4)}$. These findings support the existing health claim for prunes in the maintenance of normal bowel function.

This study was funded by the California Prune Board

1. Muller-Lissner SA, Kaatz V, Brandt W et al. (2005) Eur J Gastroenterol Hepatol 17, 109-12.

2. Attaluri A, Donahoe R, Valestin J et al. (2001) Aliment Pharmacol Ther 33, 822-8.

3. Stacewicz-Sapuntzakis M (2013) Crit Rev Food Sci Nutr 53,1277-302.

4. Cummings JH, Bingham SA, Heaton KW et al. (1992) Gastroenterology 103, 1783-9. 